\title{
TEORIA E PRÁTICA NA PESQUISA SOBRE QUALIDADE EM SERVIÇOS TURISTICOS EM PERIÓDICOS INTERNACIONAIS: UMA REVISÃO DE LITERATURA DE 2002 A 2012
}

\author{
THEORY AND PRACTICE IN RESEARCH \\ OF QUALITY IN TOURISM SERVICES IN \\ INTERNATIONAL JOURNALS: \\ A LITERATURE REVIEW OF 2002 TO 2012
}

Recebido 11-03-2013 Aceito 25-01-2014

Alexandre Meira de Vasconcelos ${ }^{1}$ Álvaro Guillermo Rojas Lezana²

\section{RESUMO}

Este artigo apresenta uma revisão de literatura sobre a qualidade em serviços turísticos baseada em artigos de periódicos em bases de dados indexadas. Os documentos foram selecionados em quinze bases, filtrados por métodos arbitrados pelos autores e 105 artigos resultantes foram lidos integralmente e avaliados pelo método de análise de conteúdo. Entre os resultados principais estão uma matriz conceitual para a qualidade de serviços turísticos e um conjunto de lacunas e oportunidades para pesquisas futuras. Os resultados mais relevantes estão (1) a definição da relação de causalidade entre a qualidade dos serviços e a lealdade do turista ao destino turístico; (2) há diferenças entre o comprador do serviço e o indivíduo que consome o serviço turístico e a literatura não faz um tratamento diferenciado (3) baixa produção teórica sobre o tema, o que abre espaço para futuras pesquisas na área; (4) a pesquisa sobre qualidade em serviços turísticos é sustentada em teorias oriundas do marketing, e carecem da contribuição de outras áreas do conhecimento; (5) o uso excessivo de modelos generalistas para medição da qualidade, tal como o SERVQUAL; (6) excesso de estudos empíricos com equívocos metodológicos provocados por amostragens cientificamente inconsistentes; (7) medição da qualidade centrada na percepção do cliente, centrada na reação imediata ao evento vivido e desconsidera os múltiplos elementos da cadeia de suprimentos do trade turístico.

Palavras-chave: turismo; qualidade em serviços; análise de conteúdo.

${ }^{1}$ Possui graduação em Engenharia de Produção pela Universidade Federal do Rio de Janeiro - UFRJ. Mestrado e doutorado em Engenharia de Produção na Universidade Federal de Santa Catarina - UFSC. Atualmente é professor assistente em Engenharia de Produção pela Universidade Federal de Mato Grosso do Sul - UFMS. Campo Grande, Mato Grosso do Sul, Brasil. Email: alexandre.meira@ufms.br

${ }^{2}$ Possui graduação em Engenharia Química pela Universidad Catolica de Valparaiso Chile. Mestrado em Engenharia da Produção pela Universidade Federal de Santa Catarina - UFSC. Doutorado em Engenharia Industrial pela Universidad Politecnica de Madrid - UPM. Atualmente é professor titular na Universidade Federal de Santa Catarina - UFSC. Florianópolis, Santa Catarina, Brasil. Email: alvaro.lezana@ ufsc.br 


\begin{abstract}
This article presents a literature review on quality in tourism services based on journal articles indexed in databases. The documents were selected in fifteen databases, filtered and 105 resulting articles were read and fully evaluated by the method of content analysis. Among the main results are a conceptual matrix for the quality of tourist services and a number of gaps and opportunities for future research. The most significant results are: (1) definition of the causal relationship between service quality and loyalty of the tourist to tourist destination; (2) the literature does not consider the differences between the client of a tour company and the tourist and they deserved treatment differentiated; (3) low theoretical discussion on the issue, which gives scope for further research in the area; (4) research on quality in tourism services is supported by theories of marketing, and require the contribution from other areas of knowledge; (5) excessive use of general models for quality measurement, such as SERVQUAL; (6) excess empirical studies with methodological mistakes caused by sampling scientifically inconsistent; (7) quality measurement focused on customer perception and immediate reaction to the event which ignores the multiple elements of the supply chain of the tourism trade.
\end{abstract}

Keywords: tourism, service quality, content analysis

\title{
1 INTRODUÇÃO
}

O objetivo deste artigo é revisar a literatura sobre qualidade de serviços turísticos. Trata-se, portanto, de um estudo teórico exploratório, com textos dos artigos avaliados por meio da análise de conteúdo. Para o alcance deste objetivo geral, fez-se a seleção de artigos em bases de dados indexadas e a análise integral dos artigos selecionados. Escolheu-se estudar a atividade turística por ser um segmento importante para a economia e por dinamizar a economia local e ter potencial para estimular o desenvolvimento socioeconômico de países pobres (RAMSARANFOWDAR, 2007; SAFFU et al., 2008; TIEDEMANN; VAN BIRGELE; SEMEIJN, 2009; AZEVEDO, et al., 2012). Entretanto, é sabido que a demanda por serviços turísticos é solicitada por uma clientela heterogênea, fragmentada e exigente (GARCÍA; PICOS, 2009), que requer qualidade. Ser competitivo, no cenário atual demanda qualidade nas operações, mas também no tratamento dos aspectos subjetivos desta qualidade, principalmente os relacionados ao turista. A organização prestadora de serviços e o destino turístico devem estar orientados para seu contentamento, deixando-o satisfeito, desejoso de retornar ou de indicar os serviços a outrem (HE; SONG, 2009; SAINAGHI, 2010). Narayan; Rajendran e Sai (2008) defendem que a oferta de serviços de qualidade como: boa logística, alimentação, hospitalidade e segurança são mais importantes que os atrativos naturais e que estão além do que Brunner-Sperdin e Peters (2009) chamaram de paradigma dos 4S (sun, sea, sand, sex).

O desempenho das empresas tem efeito direto sobre o crescimento dos negócios, criação de novos postos de trabalho e manutenção dos já existentes. A qualidade dos serviços e a consequente satisfação dos turistas são apontadas por vários especialistas como prioritárias para diferenciação e competitividade do setor, melhoria da imagem e atratividade dos destinos turísticos, conquista de novos consumidores, manutenção da fidelidade dos atuais e geração de empregos e renda para a população (ERAQI, 2006; MENG; TEPANON; UYSAL, 2008; MAK, 2011). Além disso, o turismo tem impacto sobre trabalhadores eventuais ou informais das comunidades e atrai o olhar do poder público para a melhoria de infraestrutura, preservação dos destinos turísticos e destinação de recursos. Dada a relevância do tema, resolveu-se verificar o que foi estudado nos últimos dez anos sobre a qualidade em serviços turísticos, como este conceito é tratado, quais métodos de pesquisa são priorizados, quais resultados foram relevantes e o que ainda falta ser estudado. Este artigo está dividido em quatro seções, incluindo esta introdução. A 
próxima seção trata dos métodos e técnicas de investigação para selecionar e analisar os artigos. A terceira seção versa sobre os resultados da seleção, análise e lacunas e as oportunidades para novas pesquisas. A última seção traz as conclusões para o estudo.

\section{MÉTODOS E TÉCNICAS}

O método de coleta e análise dos artigos está esquematizado na Figura 1.

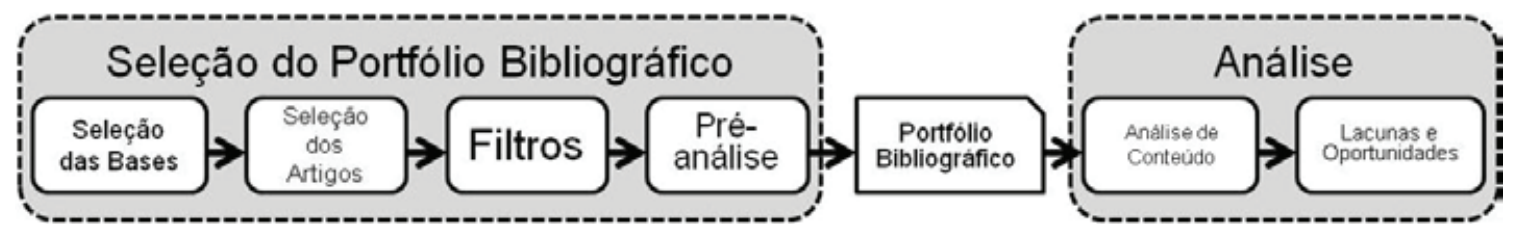

Figura 1 - Método para coleta e análise dos artigos

Fonte: Autores

Para esta pesquisa, foram selecionados artigos em quinze bases de dados indexadas (Academic Search Premier - ASP (EBSCO), ACS Journals Search, Annual Reviews, Cambridge Journals Online, Compendex (Engineering Village 2), Emerald Fulltext (Emerald), Highwire Press, IEEE Xplore, JSTOR, SAGE Journals Online, Scielo.br, ScienceDirect (Elsevier), SCOPUS (Elsevier), Web of Science, Wiley Online Library) com a busca das palavras-chave "qualidade" e "desempenho" combinadas com "turismo" nos campos título, resumo e/ou palavras-chave dos artigos. Nesta seleção, Priorizaram-se artigos científicos e de revisão e desconsiderados livros, artigos no prelo (não publicados na íntegra), editoriais, notas e literatura cinzenta em geral. Esta escolha prioriza artigos, porque estes são evidências mais atualizadas do progresso científico e se originam de um processo reconhecido no mundo acadêmico, avaliado por pares, documentado, registrado e formal (FACHIN; HILLESHEIM, 2006). O recorte temporal dos últimos dez anos (2002-2012) foi estabelecido para considerar publicações mais recentes sobre o assunto. Os artigos foram filtrados por critérios de duplicidade, alinhamento aos objetivos da pesquisa e critérios com uma abordagem qualitativa por meio da análise de conteúdo (BARDIN, 2011), utilizando os seguintes critérios arbitrados pelos autores:

a. aderência do conteúdo aos objetivos da pesquisa - este critério possibilita avaliar se o texto apresenta um estudo sobre avaliação do desempenho e/ou da qualidade nos segmentos de turismo prioritários para o Ministério do Turismo: meios de hospedagem, agências de turismo, transportadoras turísticas, organizadores de eventos (BRASIL, 2012);

b. contribuição teórico-empírica dos mesmos para a pesquisa - este critério considera os resultados obtidos e a originalidade/relevância acadêmica;

c. robustez do aporte teórico - este critério considera a qualidade e quantidade do aporte teórico apresentado pelos autores.

Cada critério recebeu pontuação em uma escala Likert de 1 a 3 e uma nota global foi atribuída a cada artigo pela soma das pontuações dos critérios. Todos os artigos com nota 1 para o critério de aderência foram eliminados e os que restaram compuseram o portfólio de artigos que foram lidos e seu conteúdo analisado na íntegra.

Para análise do conteúdo, empregou-se a lógica apresentada na Figura 2 que horizontalmente representou a leitura integral do conteúdo dos artigos e identificação das dimensões 
de análise no corpo do texto e verticalmente a análise dos excertos encontrados, por intermédio das seguintes dimensões de análise:

a. conceito de qualidade em serviços turísticos - a leitura e análise dos artigos visou compreender as óticas sob as quais este conceito é tratado na literatura, bem como correntes de interpretação e modelos conceituais empregados pelos autores;

b. métodos e processos - com o intuito de compreender os métodos de investigação, bem como os universos e amostras nas quais o estudo foi realizado;

c. resultados da pesquisa - para evidenciar os principais resultados teórico-empíricos das investigações;

d. lacunas e/ou oportunidades de pesquisa - normalmente as lacunas foram apresentadas nos artigos na forma de limitações do estudo e as oportunidades nas recomendações para estudos futuros.

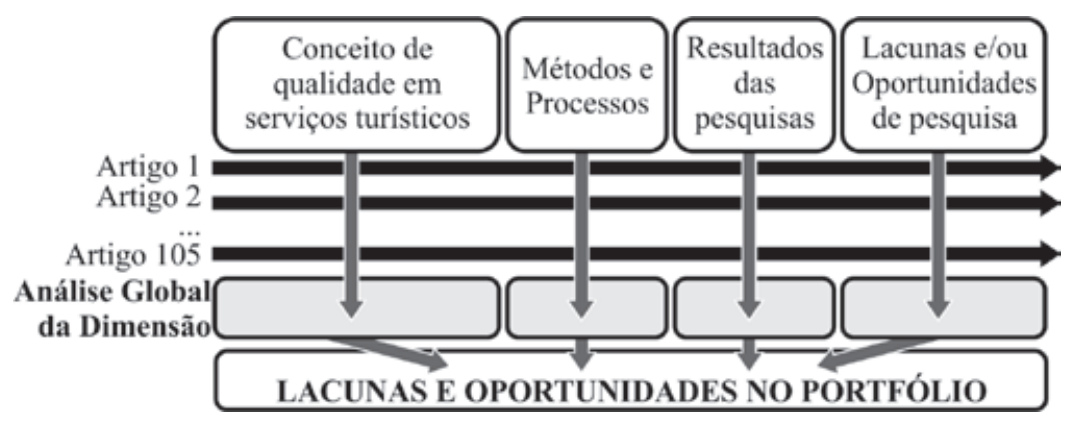

Figura 2 - Esquema da análise de conteúdo Fonte: Autores

Após a análise de todos os artigos, foi possível comparar os resultados para cada coluna do quadro de análise e extrair conclusões por critério. Foi feita uma análise geral para evidenciar as conclusões de maior destaque e possíveis lacunas/oportunidades para futuras pesquisas.

\section{RESULTADOS}

O total de 4650 artigos coletados diretamente nas bases passou por sucessivos filtros e culminou em um portfólio de 105 artigos não duplicados, com título, resumo e conteúdo alinhados aos objetivos desta pesquisa (Quadro 1).

\section{9 artigos com 9 pontos}

Bowen e Clarke (2002); Millán e Esteban (2004); Bigné et al. (2005); Bowie e Chang (2005); Haber e Reichel (2005); Kilic e Okumus (2005); Chapman e Lovell (2006); Lin (2007b); Moliner et al. (2007); Chang (2008); Gallarza e Gil (2008); Holjevac (2008); Narayan; Rajendran e Sai (2008); Nusair e Kandampully (2008); Shonk e Chelladurai (2008); Yuan e Wu (2008); Brunner-Sperdin e Peters (2009); García e Picos (2009); He e Song (2009); Wen (2009); Wooten e Norman (2009); Högström; Rosner e Gustafsson (2010); Sainaghi (2010); Salazar; Costa e Rita (2010); Wadongo et al. (2010); Moliner-Velázquez; Gil-Saura e RuizMolina (2011); Xie (2011); Kim; Ritchie e Mccormick (2012); Xiao; O'Neill e Mattila (2012)

\section{9 artigos com 8 pontos}

Juwaheer e Ross (2003); Morrison e Teixeira (2004); Gilbert e Gao (2005); Poon e Low (2005); Hwang e Lockwood (2006); Kvist e Klefsjö (2006); Benítez; Martín e Román (2007); Faullant; Matzler e Füller (2008); Meng; Tepanon e Uysal (2008); Zouni e Kouremenos (2008); Campo e Yagüe (2009); Chiu (2009); Hernández-Maestro; Muñoz-Gallego e Santos-Requejo (2009); Svari et al. (2010); Walters; Sparks e Herington

(2010); Mak (2011); Musgrave (2011); Sirirak; Islam e Khang (2011); Yang; Jou e Cheng (2011) 


\section{7 artigos com 7 pontos}

Al-Rousan e Mohamed (2010); Assaf e Cvelbar (2010); Rodríguez Del Bosque et al. (2009); Huan; Beaman e Shelby (2002); Huang (2008); Lin (2007a); Moon et al. (2011); Nadiri e Hussain (2005b); Phillips e Louvieris (2005); Presbury; Fitzgerald e Chapman (2005); Ramsaran-Fowdar (2007); Riley (2007); Tsang e Ap (2007); Tsaur e Wang (2009); Wei (2012); Ye et al. (2012); Yilmaz e Bititci (2006)

\section{5 artigos com 6 pontos}

Hudson; Hudson e Miller (2004); Nadiri e Hussain (2005a); Nearchou e Pashardes (2008); Saffu et al. (2008); Cristea (2009); Crotts et al. (2009); Teodorescu; Stancioiu e Mitu (2009); Chiu e Wu (2010); Clerides; Hsieh e Lin (2010); Pao; Wu e Pan (2010); Pyo (2010); Quintal e Polczynski (2010); Wang; Hung e Li (2011); Wu e Song (2011); Ramanathan (2012)

\section{6 artigos com 5 pontos}

Chung e Law (2003); Gu (2003); Pawitra e Tan (2003); Cândido (2005); Hai-Yan e Baum (2006); AlbaceteSáez; Fuentes-Fuentes e Lloréns-Montes (2007); Law e Bai (2008); Michopoulou e Buhalis (2008); Wang; Vela e Tyler (2008); Fleischer e Rivlin (2009); Lin; Lee e Chen (2009); Stanciu e Hapenciuc (2009); Tiedemann; Van Birgele e Semeijn (2009); Lin; Chen e Chang (2011); Sozuer (2011); Brida; Garrido e Devesa (2012)

\section{9 artigos com 4 pontos}

Coviello; Winklhofer e Hamilton (2006); Eraqi (2006); Krambia-Kapardis e Thomas (2006); Deng (2007); Golembski (2007); Chang (2009); Eraqi (2009); Fletcher; Maharaj e James (2009); Alvarez et al. (2012) Quadro 1 - Portfólio bibliográfico com pontuação

Fonte: Autores

Nesta pesquisa apresenta-se o resultado da leitura e análise do portfólio e evidenciam-se concepções teóricas, abordagens metodológicas, principais resultados e as lacunas e recomendações encontradas neste material. Em uma pesquisa mais focada, poderiam ser utilizados somente os 29 artigos mais bem pontuados. Entretanto, este artigo tem o interesse maior de compreender, em profundidade, o estado da arte sobre a qualidade em serviços turísticos e com isso resolveu-se não descartar nenhum artigo com aderência à pesquisa, mesmo os com menor pontuação.

\subsection{ANÁLISE DO CONTEÚDO DOS ARTIGOS}

Várias lacunas e oportunidades para novas pesquisas estão diluídas no texto e depois estarão condensadas em seção própria, mais adiante. Estas análises serão complementadas com outras fontes de consulta, quando houver necessidade e/ou pertinência.

3.1.1 Análise do conceito de qualidade em serviços turísticos no portfólio bibliográfico

Os principais conceitos abordados nos artigos do portfólio estão no mapa conceitual para a qualidade de serviços turísticos propostos na Figura 3 que aponta uma relação de causalidade entre eles, tendo como raiz a qualidade dos serviços. 


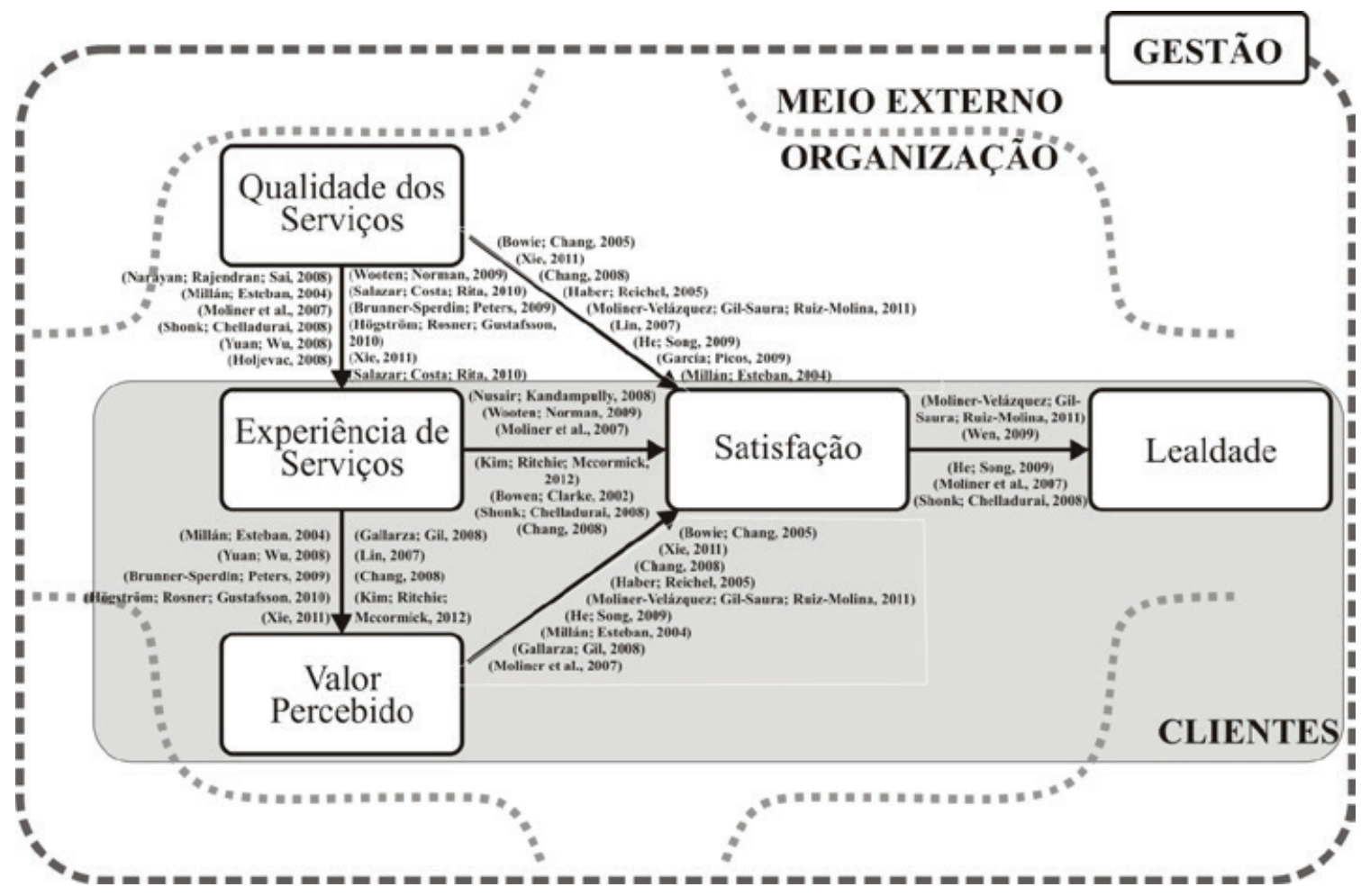

Figura 3 - Mapa conceitual sobre a qualidade em serviços turísticos

Fonte: Autores

García e Picos (2009) são taxativos em afirmar que qualidade é motivo de satisfação. A satisfação é a etapa final de um processo psicológico resultante das experiências e processos vividos na compra e consumo e depende da experiência com o uso dos produtos e/ou consumo dos serviços comparados com sentimentos anteriores (MILLÁN; ESTEBAN, 2004; MOLINER et al., 2007; NUSAIR; KANDAMPULLY, 2008). Salazar, Costa e Rita (2010) condicionam a satisfação e retenção de clientes à prestação de um serviço de excelência, bem como Haber e Reichel (2005) que apontam a elevada probabilidade de clientes satisfeitos recomendarem os serviços a outros clientes potenciais.

He e Song (2009) afirmam que a melhoria na percepção do valor e da satisfação resulta em melhor desempenho nas empresas de serviço e determinam as intenções de recompra dos turistas. A satisfação resulta em vantagem competitiva porque contribui para a intenção de recompra (YUAN; WU, 2008), porém não é somente uma expressão de uma experiência aprazível, mas o resultado da avaliação do desempenho da qualidade (BOWIE; CHANG, 2005; CHANG, 2008; SHONK; CHELLADURAI, 2008). A experiência positiva ou negativa é um processo inevitável na relação de consumo (YUAN; WU, 2008) e media a percepção de valor, porque segundo Wooten e Norman (2009) a ocorrência de um evento faz com que o indivíduo adapte a experiência à sua visão de mundo e faça uma correspondência com o que está consolidado, construa novas relações ou as revise. Chang (2008) afirma que as emoções resultantes da experiência turística contribuem para novos negócios. A experiência pressupõe a participação do turista como co-produtor do serviço, o que influencia sua percepção de valor (HÖGSTRÖM; ROSNER; GUSTAFSSON, 2010). Valor, por sua vez, não está embutido na marca ou no serviço adquirido, mas na experiência vivida no consumo (GALLARZA; GIL, 2008).

Para Chang (2008) as experiências influenciam as percepções do cliente e geram satisfação, com diferentes respostas de cada turista, visto que os objetivos, necessidades e cultura diferem um do outro e afetam a satisfação distintamente. Estas percepções têm influência direta 
sobre as intenções de recompra, porém parte desta ação é determinada pela satisfação do cliente e pela percepção de valor (HE; SONG, 2009). Kim, Ritchie e Mccormick, (2012) acreditam que as experiências memoráveis devem servir como referência ou padrão de serviço a ser entregue ao turista. Os estudos sobre a experiência, como diferenciais na prestação de serviços, é um fato que ocorre no meio acadêmico a partir do ano 2000 (REZENDE PINTO; LARA, 2009).

Moliner-Velázquez; Gil-Saura; Ruiz-Molina (2011) estabelecem que a qualidade dos serviços antecede a satisfação e a lealdade dos clientes. A lealdade é uma variável bastante estudada no turismo e está vinculada a estudos sobre o comportamento do consumidor (MOLINERVELÁZQUEZ; GIL-SAURA; RUIZ-MOLINA, 2011) e ao aumento da rentabilidade e do market share (HABER; REICHEL, 2005; GONÇALVES FILHO, et al., 2010)

Precisa-se reconhecer a qualidade do serviço como causa principal dos processos subjetivos que acontecem internamente nos clientes e, portanto, um elemento para se obter vantagem competitiva (HOLJEVAC, 2008; CAMPO; YAGÜE, 2009; ALVAREZ et al., 2012). O mapa conceitual apresenta o cliente como um elemento estratégico a ser considerado na gestão do negócio, a partir de suas predisposições e características subjetivas, pessoais e mutáveis, estabelecidas por valores culturais e influenciadas por fatores incontroláveis ou não passíveis de medição, tais como as alterações no meio-ambiente, situação político-econômica, relações de consumo, estado emocional, saúde, religiosidade e grau de suas necessidades (FINSTERWALDER; TUZOVIC, 2010).

Como a qualidade do produto turístico igualmente depende de variáveis externas, não controláveis pela organização, tais como diretrizes governamentais, incentivos, políticas de desenvolvimento, cultura local, segurança pública, presença ou ausência de atrativos naturais (NARAYAN; RAJENDRAN; SAI, 2008), o modelo da Figura 3 apresenta as fronteiras permeáveis, para confirmar que se trata de um sistema aberto e dinâmico, influenciado por diversos stakeholders, sendo o principal deles o cliente.

\subsubsection{Análise dos Métodos e Processos do portfólio bibliográfico}

Classificaram-se os artigos quanto à orientação metodológica (Tabela 1) e o resultado indica a predominância de estudos empíricos descritivos (72\%) e de estudos com abordagem quantitativa (71,5\%). Evidenciou-se que a pesquisa no setor é realizada mais em estudos exploratórios com aplicação de questionários (67,9\%) que, não obstante a sua importância científica, pouco contribuem para o aprofundamento e ou revisão das teorias que sustentam os aportes metodológicos e as análises dos dados coletados.

Tabela 1 - Orientação metodológica dos artigos

\begin{tabular}{c|c|c|c}
\cline { 2 - 4 } & DESCRITIVO & PRESCRITIVO & TOTAL \\
\hline EMPÍRICO & $\mathbf{6 8}$ & $\mathbf{2 1}$ & $\mathbf{8 9}$ \\
\hline Combinado & 3 & 3 & 10 \\
\hline Qualitativo & 7 & 3 & 73 \\
\hline Quantitativo & 58 & 15 & $\mathbf{1 6}$ \\
\hline TEÓRICO & $\mathbf{7}$ & $\mathbf{9}$ & 14 \\
\hline Qualitativo & 6 & 8 & 2 \\
\hline Quantitativo & 1 & 1 & $\mathbf{1 0 5}$ \\
\hline TOTAL & $\mathbf{7 5}$ & $\mathbf{3 0}$ & \\
\hline
\end{tabular}

Fonte: Autores 
Mesmo nos artigos teóricos deste portfólio há uma parte com estudos puramente descritivos do estado da arte $(13,3 \%)$ sem proposições de novos conceitos, modelos, métodos ou abordagens do campo do turismo (BOWEN; CLARKE, 2002; MOLINER et al., 2007; SAINAGHI, 2010). Por este motivo, entende-se que a área ainda necessita incursões teóricas mais robustas para compreender e propor novos conceitos e orientações, que abarquem as especificidades do setor de turismo, para então propor incursões empíricas sustentadas em uma teoria consistente.

Observou-se também uma frequência maior de estudos empíricos no segmento turístico de meios de hospedagem (42,9\%) em relação aos demais (Tabela 2 ). Infere-se pela natureza dos estudos e dos métodos utilizados, que o acesso às principais unidades de informação (turistas e/ou funcionários) é facilitado por estarem confinados em uma mesma estrutura física e têm disponibilidade (em alguns momentos) para serem entrevistados ou responderem a questionários.

Tabela 2 - Análise do foco do artigo, objeto e tipo de estudo

\begin{tabular}{|c|c|c|c|c|c|c|c|}
\hline \multicolumn{2}{|c|}{ FOCO DO ARTIGO } & \multirow{2}{*}{$\begin{array}{c}\text { GERAL } \\
11 \\
\end{array}$} & \multirow{2}{*}{$\begin{array}{c}\begin{array}{c}\text { Agências de } \\
\text { Turismo }\end{array} \\
5 \\
\end{array}$} & \multirow{2}{*}{\begin{tabular}{|c|}
$\begin{array}{c}\text { Meios de } \\
\text { Hospedagem }\end{array}$ \\
18 \\
\end{tabular}} & \multirow{2}{*}{\begin{tabular}{|c|}
$\begin{array}{c}\text { Organizadores } \\
\text { de Eventos }\end{array}$ \\
1 \\
\end{tabular}} & \multirow{2}{*}{\begin{tabular}{|c} 
Transportadoras \\
Turísticas \\
- \\
\end{tabular}} & \multirow{2}{*}{\begin{tabular}{|c|} 
TOTAL \\
39
\end{tabular}} \\
\hline Qualidade & Empírico & & & & & & \\
\hline em serviço & Teórico & 1 & 2 & - & 1 & - & \\
\hline \multirow{2}{*}{ Desempenho } & Empírico & 5 & 2 & 15 & - & - & \multirow{2}{*}{26} \\
\hline & Teórico & 1 & - & 3 & - & - & \\
\hline \multirow{2}{*}{$\begin{array}{l}\text { Gestão da } \\
\text { Qualidade }\end{array}$} & Empírico & 3 & 3 & 3 & - & - & \multirow{2}{*}{15} \\
\hline & Teórico & 4 & - & 2 & - & - & \\
\hline \multirow{2}{*}{ Satisfação } & Empírico & 3 & 6 & 1 & - & 1 & \multirow{2}{*}{12} \\
\hline & Teórico & 1 & - & - & - & - & \\
\hline \multirow{2}{*}{ Experiência } & Empírico & 2 & 2 & 1 & 1 & - & \multirow{2}{*}{6} \\
\hline & Teórico & - & - & - & - & - & \\
\hline \multirow{2}{*}{ Fidelização } & Empírico & 1 & - & 1 & - & - & \multirow{2}{*}{3} \\
\hline & Teórico & 1 & - & - & - & - & \\
\hline \multirow{2}{*}{ Percepção } & Empírico & - & - & 2 & - & 1 & \multirow{2}{*}{3} \\
\hline & Teórico & - & - & - & - & - & \\
\hline \multirow{2}{*}{ Expectativa } & Empírico & 1 & - & - & - & - & \multirow{2}{*}{1} \\
\hline & Teórico & - & - & - & - & - & \\
\hline \multicolumn{2}{|c|}{ TOTAL } & 35 & 20 & 45 & 3 & 2 & 105 \\
\hline
\end{tabular}

Fonte: Autores

Há um conjunto de estudos computados sob a categoria "geral" $(33,3 \%)$ que se referem a diversos elementos do destino turístico. Tratam o tema de pesquisa de forma generalista e têm como objeto o conjunto variado do trade, o setor governamental, a sociedade civil e/ou outros atores (KVIST; KLEFSJÖ, 2006; MICHOPOULOU; BUHALIS, 2008; RODRÍGUEZ DEL BOSQUE et al., 2009; SVARI et al., 2010). Alguns estudos teóricos também se enquadram como "geral" por compreenderem abordagens conceituais diversas, sem um direcionamento a um segmento específico (YILMAZ; BITITCI, 2006; RILEY, 2007; TEODORESCU; STANCIOIU; MITU, 2009). Destaca-se a baixa incidência de artigos sobre os segmentos de organizadores de eventos (SHONK; CHELLADURAI, 2008; WOOTEN; NORMAN, 2009; MOON et al., 2011) e transportadores turísticos (CHANG, 2009; BRIDA; GARRIDO; DEVESA, 2012), com estudos, em sua maioria, descritivos sobre estes segmentos.

Constatou-se a ausência de estudos teóricos sobre expectativas, experiência em serviços e percepção, e a ocorrência de um único artigo sobre fidelização e outro sobre satisfação. Todos estes construtos são importantes no âmbito da qualidade em serviços, mas pouco tem sido investigado em profundidade no campo do turismo. 
Outra evidência importante nos artigos se refere aos métodos de coleta e análise. Verificou-se a preferência pelo uso de questionários aplicados em contatos pessoais com os entrevistados ou mediados por tecnologia, principalmente por intermédio de correio eletrônico em $67,8 \%$ dos métodos de coleta e investigação adotados (Tabela 3). A soma é maior que o número de artigos analisados, porque um mesmo artigo pode usar mais de um método de análise.

A teoria clássica dos testes é o método mais usual para análise dos questionários (42\%) e outro método quantitativo, entre os mais usados, está a Estatística Descritiva (12,5\%). A Estatística Descritiva é uma análise pouco sofisticada, fornece pouca informação acerca dos dados e podem dificultar ou auxiliar a compreender os fenômenos estudados (SHEPARD, 1997). A estatística descritiva demonstrou as características demográficas da amostra (SAFFU et al., 2008; BRIDA; GARRIDO; DEVESA, 2012) ou para apresentar as frequências dos dados coletados, e medidas de centralidade ou dispersão (CLERIDES; NEARCHOU; PASHARDES, 2008; ASSAF; CVELBAR, 2010; PAO; WU; PAN, 2010). Usaram-se em geral a média, desvio-padrão e frequências absoluta e relativa para descrever os dados.

Tabela 3 - Comparação entre métodos de coleta e de análise

\begin{tabular}{|c|c|c|c|c|c|c|c|}
\hline \multirow[b]{2}{*}{ MÉTODO DE ANÁLISE } & \multicolumn{6}{|c|}{ MÉTODO DE COLETA/INVESTIGAÇÃO } & \multirow[b]{2}{*}{ TOTAL } \\
\hline & Questionário & $\begin{array}{l}\text { Pesquisa } \\
\text { teórica }\end{array}$ & Entrevista & $\begin{array}{c}\text { Dados } \\
\text { secundários }\end{array}$ & $\begin{array}{l}\text { Observação } \\
\text { participante }\end{array}$ & $\begin{array}{l}\text { Grupo } \\
\text { focal }\end{array}$ & \\
\hline Teoria clássica dos testes & 45 & & & 2 & & & 47 \\
\hline Análise de conteúdo & & 14 & 8 & 1 & 3 & 2 & 28 \\
\hline Estatística descritiva & 13 & & & 1 & & & 14 \\
\hline Lógica fuzzy & 5 & & 1 & & & & 6 \\
\hline Análise envoltória de dados & 4 & & & 1 & & & 5 \\
\hline $\begin{array}{c}\text { Modelo de equações } \\
\text { estruturais }\end{array}$ & 4 & & & & & & 4 \\
\hline Regressão linear & 1 & & & 2 & & & 3 \\
\hline $\begin{array}{l}\text { Processo de análise } \\
\text { hierárquica }\end{array}$ & 2 & & & & & & 2 \\
\hline Estudo de caso & & & 1 & & & & 1 \\
\hline Grade de repertório & 1 & & & & & & 1 \\
\hline Programação linear & 1 & & & & & & 1 \\
\hline TOTAL & 76 & 14 & 10 & 7 & 3 & 2 & 112 \\
\hline
\end{tabular}

Fonte: Autores

A Teoria Clássica dos Testes municia o pesquisador de análises mais elaboradas como a Análise Fatorial, Alfa de Cronbach, regressão logística, testes de correlação, testes de significância, testes de dimensionalidade, entre outras. Uma lacuna observada, nestas análises estatísticas, é que alguns autores realizaram testes estatísticos de significância, com amostras não aleatórias (YUAN; WU, 2008; HE; SONG, 2009; XIE, 2011), o que se configura em um erro metodológico, pois invalida por completo os estudos, mesmo quando os autores afirmam que a amostra analisada impossibilita a generalização dos resultados. Entre os estudos com abordagem qualitativa, a preferência foi pela técnica de análise de conteúdo (25\%), principalmente nos estudos teóricos e nas análises de entrevistas.

Observou-se no portfólio, que alguns instrumentos e métodos foram elaborados, modificados ou re-significados, para uso de gestão da qualidade em serviços turísticos. Na literatura, também são observados modelos de avaliação oriundos de pesquisas prescritivas que ofereceram alguma inovação ou abordagem diferenciada (BENÍTEZ; MARTíN; ROMÁN, 2007; HO; LEE, 2007; LIN, 2007a; WANG et al., 2007). Ainda assim, constatou-se que foram preparados para um segmento específico do setor de turismo, e quando tratam do setor como um todo, utiliza-se de construtos generalistas, aplicáveis a qualquer área ou atividade econômica. 


\subsubsection{Análise dos Resultados das pesquisas do portfólio bibliográfico}

As dimensões de análise "validadas" nos artigos, por meio de estatísticas, não podem em sua maioria ser generalizadas por problemas metodológicos, conforme já descrito. Tais dimensões, embora inconclusivas, apontam para uma possível incompletude das cinco dimensões de avaliação da qualidade de serviços propostas por Parasuraman; Zeithaml e Berry (1988): tangibilidade, confiabilidade, responsividade, garantia e empatia. Outras dimensões foram evidenciadas no portfólio tais como a responsabilidade socioambiental (HOLJEVAC, 2008; MUSGRAVE, 2011; SOZUER, 2011), a orientação estratégica para o mercado (HERNÁNDEZ-MAESTRO; MUÑOZ-GALLEGO; SANTOS-REQUEJO, 2009; TIEDEMANN; VAN BIRGELE; SEMEIJN, 2009), gestão da experiência (YUAN; WU, 2008; BRUNNER-SPERDIN; PETERS, 2009; KIM; RITCHIE; MCCORMICK, 2012), liderança (BOWIE; CHANG, 2005; HAI-YAN; BAUM, 2006; HWANG; LOCKWOOD, 2006), entre outras não previstas no trabalho seminal. Outra crítica aos modelos de avaliação é a supervalorização de resultados financeiros em detrimento dos não financeiros, relativos à qualidade da prestação do serviço, clientes, pessoas, inovação, entre outros (KRAMBIA-KAPARDIS; THOMAS, 2006; HUANG, 2008; WADONGO et al., 2010).

Como já assinalado, os estudos sobre qualidade em organizadores de eventos e em transportadores turísticos mostrou-se incipiente no portfólio bibliográfico, sendo os estudos mais promissores sobre agências de turismo e meios de hospedagem. Os estudos sobre as agências de turismo concluem que a qualidade dos serviços é um elemento importante para o desenvolvimento do setor, na intermediação entre o turista e o destino turístico. Entretanto, exigem operações complexas que envolvem uma elevada interação com o cliente, pouca margem na negociação de valores, venda de produtos e serviços de uma cadeia muito diversa e fragmentada. Destaca-se aqui a ameaça das tecnologias de informação e comunicação que, em alguns casos, torna a intermediação da agência desnecessária, como exemplo, as vendas online de passagens e de acomodações, por meio de negociações diretas por meio de redes sociais, entre outras (BOWEN; CLARKE, 2002; BIGNÉ et al., 2005). Sendo um dos principais pontos de partida do fluxo do serviço turístico, a agência de turismo vende serviços, que muitas vezes o cliente nunca adquiriu e tampouco sabe o que vai acontecer no destino. Assim, as informações devem ser claras (CHANG, 2008) e o staff da agência deve assumir a função de consultores de viagem, com capacidade de oferecer pacotes adequados a cada perfil do consumidor, personalizando o atendimento (MILLÁN; ESTEBAN, 2004; ALBACETE-SÁEZ; FUENTES-FUENTES; LLORÉNS-MONTES, 2007; WANG; VELA; TYLER, 2008), sob pena de gerar insatisfação do cliente, quando este fizer contato real com o destino (MOLINER et al., 2007).

Com relação aos meios de hospedagem, os resultados apontaram que há diferenças na avaliação da qualidade em hotéis, conforme o tipo de usuário e suas características demográficas e culturais (GARCíA; PICOS, 2009) e por este motivo Bowen e Clarke (2002) criticam os instrumentos de avaliação da qualidade autoadministrados comumente encontrados em hotéis, pousadas e afins. Os atributos não consideram a experiência turística, os afetos e emoções associados e normalmente são preenchidos de forma não sistemática, gerando resultados contaminados pela falta de vontade e antipatia do turista em respondê-los. A literatura também aponta a importância dos recursos humanos representada pela empatia, responsividade, responsabilidade, conhecimento, capacidade profissional, presteza, interação com funcionários e com outros turistas, entre outros, como fatores que influenciam o estado emocional de quem recebe o serviço. Por ser notoriamente uma atividade experiencial e emocional, a avaliação da qualidade do serviço por meio do turista requer a inclusão de aspectos emocionais e comportamentais (LIN, 2007a; BRUNNER-SPERDIN; PETERS, 2009). 
Tabela 4 - Tipo de coleta de dados por unidade de informação

\begin{tabular}{|c|c|c|c|c|c|c|}
\hline Unidade de Informação & Survey & $\begin{array}{c}\text { Pesquisa } \\
\text { Teórica }\end{array}$ & Entrevista & $\begin{array}{c}\text { Dados } \\
\text { Secundários }\end{array}$ & $\begin{array}{c}\text { Observação } \\
\text { Participante }\end{array}$ & Total \\
\hline turista & 44 & & & & 2 & 46 \\
\hline gestor & 23 & & 6 & & 1 & 30 \\
\hline produção científica & & 14 & & & & 14 \\
\hline dados secundários & & & & 5 & & 5 \\
\hline website & 2 & & & 2 & & 4 \\
\hline colaboradores & 3 & & & & & 3 \\
\hline especialistas & 1 & & 1 & & & 2 \\
\hline operador de turismo & 1 & & & & & 1 \\
\hline Total & $\mathbf{7 4}$ & $\mathbf{1 4}$ & $\mathbf{7}$ & $\mathbf{7}$ & $\mathbf{3}$ & $\mathbf{1 0 5}$ \\
\hline
\end{tabular}

Fonte: Autores

\subsubsection{Análise das lacunas e oportunidades de pesquisa do portfólio bibliográfico}

Inúmeros autores dos artigos do portfólio apresentaram a impossibilidade de generalização dos resultados como principal limitação e que resultam em trabalhos mais exploratórios que conclusivos (HE; SONG, 2009). Apontam também a necessidade de estudos mais aprofundados, com amostras mais representativas dos objetos de estudo. Os empecilhos de maior destaque nos artigos são:

a. amostra por conveniência, não aleatória, (YUAN; WU, 2008; SALAZAR; COSTA; RITA, 2010; KIM; RITCHIE; MCCORMICK, 2012; XIAO; O'NEILL; MATTILA, 2012) ou insuficiente (LIN, 2007b; MOLINER et al., 2007; CHANG, J. C., 2008). Considerando-se que os resultados numéricos (escores) de avaliações da qualidade são oriundos de populações com distribuições normais, as amostras devem ser obrigatoriamente aleatórias e independentes (BERENSON et al., 2005; BARBETTA; REIS; BORNIA, 2010);

b. estudo limitado a uma região (KILIC; OKUMUS, 2005), tipo de negócio (HÖGSTRÖM; ROSNER; GUSTAFSSON, 2010) ou tamanho da organização (NUSAIR; KANDAMPULLY, 2008). Sainaghi (2010) aponta o problema da regionalização dos estudos como limitador da generalização e propõe a identificação de fatores de sucesso aplicáveis a contextos distintos;

c. recorte temporal, muitas vezes por pesquisas conduzidas em dados secundários que estão disponíveis ao pesquisador (GALLARZA; GIL, 2008; BRUNNER-SPERDIN; PETERS, 2009).

Outros fatores de destaque, nas limitações dos estudos, são os modelos de análise e a dimensionalidade dos construtos, pois nem todas as dimensões de análise são relevantes. Xie (2011) observa que a construção de uma escala para avaliação da qualidade percebida, em uma ampla linha de serviços turísticos, ainda requer um esforço de pesquisa considerável. Na mesma linha de raciocínio, Brunner-Sperdin e Peters (2009) estudaram a experiência de serviços e afirmam que a literatura sobre gestão não apresenta um modelo convincente para avaliar a qualidade de uma experiência. Yuan e Wu (2008) estimulam estudos empíricos sobre marketing experiencial, visto que se trata de um conceito novo no meio acadêmico. Para resolver esta e outras situações, a literatura sugere a criação de modelos mais amplos de avaliação (BIGNÉ et al., 2005; NARAYAN; RAJENDRAN; SAI, 2008; WADONGO et al., 2010), multidimensionais (GALLARZA; GIL, 2008), com o uso de abordagens qualitativas na sua construção (SHONK; CHELLADURAI, 2008; YUAN; WU, 2008) e com escalas de medição adequadas (GARCÍA; PICOS, 2009). Os autores pro- 
põem a inclusão de novos construtos variados nas avaliações da qualidade, tais como a imagem (SALAZAR; COSTA; RITA, 2010); efeitos gerados por fatores externos (SAINAGHI, 2010); confiança entre clientes e operadores do setor (WEN, 2009); indicadores comportamentais e atitudinais (MOLINER-VELÁZQUEZ, 2011); entre outros.

\subsection{Lacunas e oportunidades de pesquisa}

Nesta seção apresenta-se um resumo com as principais lacunas e oportunidades detectadas no estudo teórico, citadas a seguir.

O conceito de turista, evidenciado na literatura, o coloca em uma posição de consumidor do produto turístico e desconsidera outras relações de consumo na cadeia de fornecimento do turismo e que podem ser incluídas no âmbito da qualidade. $O$ turista (consumidor) e o cliente não podem ser confundidos. O primeiro influencia a decisão de compra por meio de suas necessidades e o segundo realiza o desembolso. Em algumas situações, estes personagens podem ser a mesma pessoa, mas a tratativa deve ser diferenciada para cada papel exercido.

A baixa produção científica teórica, sobre a qualidade em serviços turísticos, gera a oportunidade para o meio acadêmico investigar o assunto em profundidade, e dele gerar novos conceitos e abordagens, em estudos aprofundados da literatura e da práxis por meio de métodos de pesquisa combinados (qualitativos e quantitativos) ou isolados, e por uma análise de dados estatisticamente adequada, quando a abordagem quantitativa for escolhida;

Os estudos sobre a qualidade e/ou desempenho no setor turístico são ancorados em teorias oriundas do marketing, focadas na percepção do cliente. Os trabalhos seminais não foram e nem podem ser desconsiderados para a consecução de uma teoria sobre o assunto, porém devem buscar contribuições de outras áreas, tais como as Engenharias, Geografia, História, Ciências Biológicas, Antropologia, Psicologia, Artes, entre outras. Isto conferiria um caráter multidisciplinar que pode contribuir para o desenvolvimento da qualidade no setor, melhoria do desempenho e crescimento da atividade turística, incorporando expertises até o momento não trabalhadas ou tratadas de forma insipiente ou pouco profissional. O conceito de qualidade em serviços, observado na literatura, não difere atualmente das abordagens seminais e está fortemente ancorado na percepção dos clientes da dicotomia expectativa-desempenho. Essa constatação pode estar ancorada na formação e atuação em Marketing dos autores das principais referências ainda cultuados no meio acadêmico, quando se fala em qualidade de serviços: Richard Oliver, Christian Grönroos, A. Parasuraman, Valarie Zeitham, entre outros. Por sua formação, estes autores têm um olhar mais atento aos aspectos mercadológicos e na qualidade percebida e menos na gestão da qualidade e das operações de serviços;

Evidenciou-se a ausência de parâmetros que considerem a amplitude e o alcance da qualidade no setor de turismo, em meio ao uso predominante de modelos generalistas, de avaliação de serviços, para avaliar o setor turístico (principalmente o SERVQUAL) ou modelos específicos para determinado segmento tais como hotéis, companhias aéreas, entre outros.

A produção científica sobre a qualidade em serviços turísticos ainda é incipiente, o que pode significar um campo fértil para estudos. Além disso, é um ramo da economia com elevado potencial de crescimento no Brasil, com uma cadeia de suprimentos complexa e um número elevado de operações.

Os estudos sobre qualidade no setor turístico são em sua maioria voltados a avaliar a satisfação ou percepção do cliente e menos a gestão do negócio. Poucos estudos consideram os aspectos estratégicos e operacionais, pois se concluiu, a partir do referencial teórico, que a satisfação, a percepção, experiências de serviço memoráveis e a fidelização são consequências da qualidade dos serviços prestados. 
Inúmeros estudos relatam a impossibilidade de generalização dos resultados por conta de amostras inadequadas ou por focar somente um destino ou um grupo de empresas. Pesquisadores devem refletir e suas escolhas metodológicas e, optando por abordagens quantitativas, devem seguir o rigor dos métodos estatísticos tanto na amostragem quanto na análise dos resultados.

A qualidade do serviço é comumente medida pela percepção relatada pelo cliente, mesmo com a complexidade da cadeia do turismo que envolve múltiplos serviços. Esta forma de medição resulta em análises subjetivas e que não subsidiam o gestor para a tomada de decisão e operacionalização das melhorias da qualidade. Deve-se medir a qualidade do serviço por meio de dimensões tangíveis e mensuráveis, focadas na gestão do negócio tais como as relacionadas à infraestrutura, recursos humanos, operações, relacionamento com clientes, entre outras.

\section{CONCLUSÕES}

As lacunas e oportunidades de pesquisa sobre qualidade, em serviços turísticos, foram identificadas por meio de uma pesquisa teórica sistemática em profundidade e espera-se que os resultados, apresentados na seção anterior, estimulem outros pesquisadores a se aprofundarem nos temas que são passíveis de investigação e que cuidem dos equívocos metodológicos assinalados.

O mapa conceitual para a qualidade em serviços turísticos evidenciou a relação de causalidade entre a qualidade de serviços e a lealdade com o destino turístico, mediadas pela experiência de serviços, o valor percebido e a satisfação, devendo ser o cliente o foco estratégico da organização. Entre estes conceitos destaca-se a experiência em serviços, que carece de maior interesse da academia, tanto do ponto de vista teórico quanto empírico, frente ao forte caráter vivencial da atividade turística.

Este trabalho igualmente conclui que os estudos sobre qualidade em serviços turísticos carecem de maior adequação e rigor metodológicos, de um aporte teórico mais consistente e que sejam aplicados nos mais diferentes segmentos deste setor da economia e no destino turístico como um todo. A falta de método científico resultou em trabalhos com problemas relacionados, por exemplo, a questionamentos quanto à dimensionalidade dos construtos e à impossibilidade de generalização dos resultados.

Esta pesquisa não poderia ser realizada sem o auxílio computacional, devido ao grande número de artigos trabalhados. Foram lidos, conforme já dito, o título e resumo de 4096 artigos, o conteúdo parcial de 179 e o conteúdo integral de 105 deles, o que seria uma tarefa bastante complicada sem a catalogação intermediada por softwares.

As limitações deste trabalho repousam principalmente no viés da análise, centrada na figura dos autores, o que é impossível desvencilhar em um trabalho desta natureza. Para tanto, buscou-se rigor metodológico na coleta e análise dos artigos para diminuir este viés. Outra limitação é a de que foram consultadas somente bases de dados com acesso pela Capes e a artigos com textos completos, que podem gerar alguma perda relevante de conteúdo. 


\section{REFERÊNCIAS}

ALBACETE-SÁEZ, C. A.; FUENTES-FUENTES, M. M.; LLORÉNS-MONTES, F. J. Service quality measurement in rural accommodation. Annals of Tourism Research, v. 34, n. 1, p. 45-65, 2007.

AL-ROUSAN, M. R.; MOHAMED, B. Customer loyalty and the impacts of service quality: The case of five star hotels in Jordan. World Academy of Science, Engineering and Technology, v. 67, p. 216-222, 2010.

ALVAREZ, M. J. et al. Quality management in hotels in the Basque Country. International Journal of Quality and Service Sciences, v. 4, n. 1, p. 51-60, 2012.

ASSAF, A. G.; CVELBAR, L. K. The performance of the Slovenian hotel industry: Evaluation post-privatisation. International Journal of Tourism Research, v. 12, n. 5, p. 462-471, 2010.

AZEVEDO, M. S., MARTINS, C. B., PIZZINATTO, N. K., FARAH, O. E.. Segmentação no setor turístico: o turista LGBT de São Paulo. Revista de Administração da UFSM, v. 5, n. 3, p. 493506, 2012.

BARBETTA, P. A.; REIS, M. M.; BORNIA, A. C. Estatística para cursos de engenharia $e$ informática. 3a ed. São Paulo-SP: Atlas, 2010.

BARDIN, L. Análise de Conteúdo. São Paulo: Edições 70, 2011.

BENÍTEZ, J. M.; MARTÍN, J. C.; ROMÁN, C. Using fuzzy number for measuring quality of service in the hotel industry. Tourism Management, v. 28, n. 2, p. 544-555, 2007.

BERENSON, M. L. et al. Estatística: teoria $e$ aplicações usando microsoft excel em português. Rio de Janeiro: LTC Livros Técnicos e Científicos Editora 2005.

BIGNÉ, J. E. et al. Quality Market Orientation: Tourist Agencies' Perceived Effects. Annals of Tourism Research, v. 32, n. 4, p. 1022-1038, 2005.
BOWEN, D.; CLARKE, J. Reflections on tourist satisfaction research: Past, present and future. Journal of Vacation Marketing, v. 8, n. 4, p. 297-308, September 1, 2002.

BOWIE, D.; CHANG, J. C. Tourist satisfaction: A view from a mixed international guided package tour. Journal of Vacation Marketing, $v$. 11, n. 4, p. 303-322, October, 2005.

BRASIL. CADASTUR: Sistema de Cadastro de pessoas físicas e jurídicas que atuam no setor do turismo. Brasília-DF, 2012. Disponível em: <http://www.cadastur.turismo.gov.br/ cadastur/index.action >. Acesso em:10 de Março de 2012.

BRIDA, J. G.; GARRIDO, N.; DEVESA, M. J. S. Cruise passengers' satisfaction: Cartagena de Indias. Benchmarking: An International Journal, v. 19, n. 1, p. 52-69, 2012.

BRUNNER-SPERDIN, A.; PETERS, M. What Influences Guests' Emotions? The Case of High-quality Hotels. International Journal of Tourism Research, v. 11, n. 2, p. 171-183, 2009.

CAMPO, S.; YAGÜE, M. J. Exploring nonlinear effects of determinants on tourists' satisfaction. International Journal of Culture, Tourism and Hospitality Research, v. 3, n. 2, p. 127-138, 2009.

CÂNDIDO, C. J. F. Service quality strategy implementation: A model and the case of the Algarve hotel industry. Total Quality Management and Business Excellence, v. 16, n. 1, p. 3-14, 2005.

CHANG, J.C. Taiwanese tourists' perceptions of service quality on outbound guided package tours: A qualitative examination of the SERVQUAL dimensions. Journal of Vacation Marketing, v. 15, n. 2, p. 165-178, April, 2009.

Tourists' Satisfaction Judgments: An Investigation of Emotion, Equity, and Attribution. Journal of Hospitality \& Tourism Research, v. 32, n. 1, p. 108-134, February 1, 2008. 
CHAPMAN, J. A.; LOVELL, G. The competency model of hospitality service: why it doesn't deliver. International Journal of Contemporary Hospitality Management, v. 18, n. 1, p. 78-88, 2006.

CHEN, C. F.; CHEN, F. S. Experience quality, perceived value, satisfaction and behavioral intentions for heritage tourists. Tourism Management, v. 31, n. 1, p. 29-35, Feb 2010.

CHIU, C.-K. Understanding relationship quality and online purchase intention in e-tourism: $\mathrm{A}$ qualitative application. Quality \& Quantity, v. 43, n. 4, p. 669-675, 2009.

CHIU, Y. H.; WU, M. F. Performance evaluation of international tourism hotels in Taiwan Application of context-dependent DEA. INFOR, v. 48, n. 3, p. 155-170, 2010.

CHUNG, T.; LAW, R. Developing a performance indicator for hotel websites. International Journal of Hospitality Management, v. 22, n. 1, p. 119-125, 2003.

CLERIDES, S.; NEARCHOU, P.; PASHARDES, P. Intermediaries as quality assessors: Tour operators in the travel industry. International Journal of Industrial Organization, v. 26, n. 1, p. 372-392, 2008.

COVIELLO, N.; WINKLHOFER, H.; HAMILTON, K. Marketing Practices and Performance of Small Service Firms. Journal of Service Research, v. 9, n. 1, p. 38-58, August, 2006.

CRISTEA, A. A. The reasssesment of the service quality system within the romanian hotel industry - A premise for increasing the competition of the tourist offer. Quality Management in Services, v. 26, n. 1, p. 451461, 2009.

CROTTS, J. C.; FORD, R. C.; HEUNG, V. C. S.; NGAI, E. W. T. Organizational alignment and hospitality firm performance. International Journal of Culture, Tourism and Hospitality Research, v. 3, n. 1, p. 3-12, 2009.
DENG, W. Using a revised importanceperformance analysis approach: The case of Taiwanese hot springs tourism. Tourism Management, v. 28, n. 5, p. 1274-1284, 2007.

ERAQI, M. I. Integrated quality management and sustainability for enhancing the competitiveness of tourism in Egypt. International Journal of Services and Operations Management, v. 5, n. 1, p. 14-28, 2009.

Tourism services quality (TourServQual) in Egypt: The viewpoints of external and internal customers. Benchmarking: An International Journal, v. 13, n. 4, p. 469-492, 2006.

FACHIN, G. R. B.; HILLESHEIM, A. I. A. Periódico científico: padronização e organização. Florianópolis: Editora da UFSC, 2006.

FAULLANT, R.; MATZLER, K.; FÜLLER, J. The impact of satisfaction and image on loyalty: the case of Alpine ski resorts. Managing Service Quality, v. 18, n. 2, p. 163-178, 2008.

FINSTERWALDER, J.; TUZOVIC, S. Quality in group service encounters $A$ theoretical exploration of the concept of a simultaneous multi-customer co-creation process. Managing Service Quality, v. 20, n. 2, p. 109-122, 2010.

FLEISCHER, A.; RIVLIN, J. More or Better?: Quantity and Quality Issues in Tourism Consumption. Journal of Travel Research, v. 47, n. 3, p. 285-294, 2009.

FLETCHER, S. M.; MAHARAJ, S. R.; JAMES, K. Description of the Food Safety System in Hotels and How It Compares With HACCP Standards. Journal of Travel Medicine, v. 16, n. 1, p. 35-41, 2009.

GALLARZA, M. G.; GIL, I. The concept of value and its dimensions: a tool for analysing tourism experiences. Tourism Review, v. 63, n. 3, p. 4-20, 2008. 
GARCÍA, M. L.; PICOS, A. P. La calidad percibida como determinante de tipologías de clientes y su relación con la satisfacción: Aplicación a los servicios hoteleros. Revista Europea de Dirección y Economía de la Empresa, v. 18, n. 3, p. 189-210, 2009.

GILBERT, D.; GAO, Y. A Failure of UK Travel Agencies to Strengthen Zones of Tolerance. Tourism and Hospitality Research, v. 5, n. 4, p. 306-321, August, 2005.

GOLEMBSKI, G. The impact of modern management methods on hotel operational performance. Tourism Review, v. 62, n. 2, p. 31-36, 2007.

GONÇALVES FILHO, C. et al. Lealdade intencional e comportamento: influências contextuais e individuais no setor de telefonia móvel pós-paga. Revista de Administração da UFSM, v. 3, n. 3, p. 441-458, 2010.

GU, Z. The Chinese lodging industry: problems and solutions. International Journal of Contemporary Hospitality Management, v. 15, n. 7, p. 386-392, 2003.

HABER, S.; REICHEL, A. Identifying performance measures of small ventures - The case of the tourism industry. Journal of Small Business Management, v. 43, n. 3, p. 257-286, 2005.

HAI-YAN, K.; BAUM, T. Skills and work in the hospitality sector: The case of hotel front office employees in China. International Journal of Contemporary Hospitality Management, v. 18, n. 6, p. 509-518, 2006.

HE, Y.; SONG, H. A Mediation Model of Tourists' Repurchase Intentions for Packaged Tour Services. Journal of Travel Research, v. 47, n. 3, p. 317-331, February 1, 2009.

HERNÁNDEZ-MAESTRO， R. M.; MUÑOZGALLEGO, P. A.; SANTOS-REQUEJO, L. SmallBusiness Owners' Knowledge and Rural Tourism Establishment Performance in Spain. Journal of Travel Research, v. 48, n. 1, p. 58-77, 2009.
HO, C. I.; LEE, Y. L. The development of an e-travel service quality scale. Tourism Management, v. 28, n. 6, p. 1434-1449, 2007.

HÖGSTRÖM, C.; ROSNER, M.; GUSTAFSSON, A. How to create attractive and unique customer experiences: An application of Kano's theory of attractive quality to recreational tourism. Marketing Intelligence \& Planning, v. 28, n. 4, p. 385-402, 2010.

HOLJEVAC, I. A. Business ethics in tourism - As a dimension of TQM. Total Quality Management and Business Excellence, v. 19, n. 10, p. 10291041, 2008.

HSIEH, L. F.; LIN, L. H. A performance evaluation model for international tourist hotels in TaiwanAn application of the relational network DEA. International Journal of Hospitality Management, v. 29, n. 1, p. 14-24, Mar 2010.

HUAN, T. C.; BEAMAN, J.; SHELBY, L. B. Using action-grids in tourism management. Tourism Management, v. 23, n. 3, p. 255-264, 2002.

HUANG, L. Strategic orientation and performance measurement model in Taiwan's travel agencies. Service Industries Journal, v. 28, n. 10, p. 1357-1383, 2008.

HUDSON, S.; HUDSON, P.; MILLER, G. A. The Measurement of Service Quality in the Tour Operating Sector: A Methodological Comparison. Journal of Travel Research, v. 42, n. 3, p. 305-312, 2004.

HWANG, L.-J. J.; LOCKWOOD, A. Understanding the challenges of implementing best practices in hospitality and tourism SMEs. Benchmarking: An International Journal, v. 13, n. 3, p. 337-354, 2006.

JUWAHEER, T. D.; ROSS, D. L. A study of hotel guest perceptions in Mauritius. International Journal of Contemporary Hospitality Management, v. 15, n. 2, p. 105-115, 2003.

KILIC, H.; OKUMUS, F. Factors influencing productivity in small island hotels: Evidence 
from Northern Cyprus. International Journal of Contemporary Hospitality Management, v. 17, n. 4, p. 315-331, 2005.

KIM, J. H.; RITCHIE, J. R. B.; MCCORMICK, B. Development of a Scale to Measure Memorable Tourism Experiences. Journal of Travel Research, v. 51, n. 1, p. 12-25, 2012.

KRAMBIA-KAPARDIS, M.; THOMAS, A. Hospitality industry in Cyprus: the significance of intangibles. International Journal of Contemporary Hospitality Management, v. 18, n. 1, p. 6-24, 2006.

KVIST, A.-K. J.; KLEFSJÖ, B. Which service quality dimensions are important in inbound tourism?: A case study in a peripheral location. Managing Service Quality, v. 16, n. 5, p. 520537, 2006.

LAW, R.; BAI, B. How do the preferences of online buyers and browsers differ on the design and content of travel websites? International Journal of Contemporary Hospitality Management, v. 20, n. 4, p. 388-400, 2008.

LIN, C.-T.; LEE, C.; CHEN, W. Y. Using fuzzy analytic hierarchy process to evaluate service performance of a travel intermediary. Service Industries Journal, v. 29, n. 3, p. 281-296, 2009.

LIN, L.-Z.; CHEN, W.-C.; CHANG, T.-J. Using FQFD to analyze island accommodation management in fuzzy linguistic preferences. Expert Systems with Applications, v. 38, n. 6, p. 7738-7745, 2011.

LIN, W.B. An empirical of service quality model from the viewpoint of management. Expert Systems with Applications, v. 32, n. 2, p. 364375, 2007a.

The exploration of customer satisfaction model from a comprehensive perspective. Expert Systems with Applications, v. 33 , n. 1, p. 110-121, 2007b.

MAK, B. L. M. ISO certification in the tour operator sector. International Journal of
Contemporary Hospitality Management, v. 23, n. 1, p. 115-130, 2011.

MASON, P.; AUGUSTYN, M.; SEAKHOA-KING, A. Exploratory Study in Tourism: Designing an Initial, Qualitative Phase of Sequenced, Mixed Methods Research. International Journal of Tourism Research, v. 12, n. 5, p. 432-448, SepOct 2010.

MENG, F.; TEPANON, Y.; UYSAL, M. Measuring tourist satisfaction by attribute and motivation: The case of a nature-based resort. Journal of Vacation Marketing, v. 14, n. 1, p. 41-56, January, 2008.

MICHOPOULOU, E.; BUHALIS, D. Performance measures of net-enabled hypercompetitive industries: The case of tourism. International Journal of Information Management, v. 28, n. 3, p. 168-180, 2008.

MILLÁN, Á.; ESTEBAN, A. Development of a multiple-item scale for measuring customer satisfaction in travel agencies services. Tourism Management, v. 25, n. 5, p. 533-546, 2004.

MOLINER, M. A. et al. Relationship Quality with a Travel Agency: The Influence of the Postpurchase Perceived Value of a Tourism Package. Tourism and Hospitality Research, v. 7, n. 3-4, p. 194-211, September 1, 2007.

MOLINER-VELÁZQUEZ, B.; GIL-SAURA, I.; RUIZMOLINA, M.-E. Conceptualizing and measuring loyalty: Towards a conceptual model of tourist loyalty antecedents. Journal of Vacation Marketing, v. 17 , n. 1, p. 65-81, January 1, 2011.

MOON, K. S. et al. The influence of consumer's event quality perception on destination image. Managing Service Quality, v. 21, n. 3, p. 287303, 2011.

MORRISON, A.; TEIXEIRA, R. Small business performance: a tourism sector focus. Journal of Small Business and Enterprise Development, v. 11, n. 2, p. 166-173, 2004. 
MUSGRAVE, J. Moving towards responsible events management. Worldwide Hospitality and Tourism Themes, v. 3, n. 3, p. 258-274, 2011.

NADIRI, H.; HUSSAIN, K. Diagnosing the zone of tolerance for hotel services. Managing Service Quality, v. 15, n. 3, p. 259-277, 2005a.

Perceptions of service quality in North Cyprus hotels. International Journal of Contemporary Hospitality Management, v. 17, n. 6 , p. 469-480, 2005b.

NARAYAN, B.; RAJENDRAN, C.; SAI, L. P. Scales to measure and benchmark service quality in tourism industry: A second-order factor approach. Benchmarking: An International Journal, v. 15, n. 4, p. 469-493, 2008.

NUSAIR, K.; KANDAMPULLY, J. The antecedents of customer satisfaction with online travel services: A conceptual model. European Business Review, v. 20, n. 1, p. 4-19, 2008.

PAO, H. W.; WU, H. L.; PAN, W. H. How does a new institution spread? Travel agencies' adoption of the service quality assurance programme. Service Industries Journal, v. 30, n. 7, p. 1047-1061, 2010.

PARASURAMAN, A.; ZEITHAML, V.; BERRY, L. L. SERVQUAL: A multiple-item scale for measuring consumer perceptions of service quality. Journal of Retailing, v. 64, n. 1, p. 1240, 1988.

PAWITRA, T. A.; TAN, K. C. Tourist satisfaction in Singapore - a perspective from Indonesian tourists. Managing Service Quality, v. 13, n. 5, p. 399-411, 2003.

PHILLIPS, P.; LOUVIERIS, P. Performance Measurement Systems in Tourism, Hospitality, and Leisure Small Medium-Sized Enterprises: A Balanced Scorecard Perspective. Journal of Travel Research, v. 44, n. 2, p. 201-211, 2005.

POON, W.-C.; LOW, K. L.-T. Are travellers satisfied with Malaysian hotels? International Journal of
Contemporary Hospitality Management, v. 17, n. 3, p. 217-227, 2005.

PRESBURY, R.; FITZGERALD, A.; CHAPMAN, R. Impediments to improvements in service quality in luxury hotels. Managing Service Quality, v. 15, n. 4, p. 357-373, 2005.

PYO, S. Measuring tourism chain performance. Service Industries Journal, v. 30, n. 10, p. 16691682, 2010.

QUINTAL, V. A.; POLCZYNSKI, A. Factors influencing tourists' revisit intentions. Asia Pacific Journal of Marketing and Logistics, v. 22, n. 4, p. 554-578, 2010.

RAMANATHAN, R. An exploratory study of marketing, physical and people related performance criteria in hotels. International Journal of Contemporary Hospitality Management, v. 24, n. 1, p. 44-61, 2012.

RAMSARAN-FOWDAR, R. R. Developing a service quality questionnaire for the hotel industry in Mauritius. Journal of Vacation Marketing, v. 13, n. 1, p. 19-27, January, 2007.

REZENDE PINTO, M.; LARA, J. E. O que se publica sobre comportamento do consumidor no Brasil, afinal? Revista de Administração da UFSM, v. 1, n. 3, 2009.

RILEY, M. Role interpretation during service encounters: A critical review of modern approaches to service quality management. International Journal of Hospitality Management, v. 26, n. 2, p. 409-420, 2007.

RODRÍGUEZ DEL BOSQUE, l. et al. A framework for tourist expectations. International Journal of Culture, Tourism and Hospitality Research, v. 3, n. 2, p. 139-147, 2009.

SAFFU, K. OBENG APORI, S., ELIJAH-MENSAH, A., \& AHUMATAH, J. The contribution of human capital and resource-based view to small- and medium-sized tourism venture performance in Ghana. International Journal of Emerging Markets, v. 3, n. 3, p. 268-284, 2008. 
SAINAGHI, R. A meta-analysis of hotel performance. Continental or worldwide style? Tourism Review, v. 65, n. 3, p. 46-69, 2010.

SALAZAR, A.; COSTA, J.; RITA, P. A service quality evaluation scale for the hospitality sector: Dimensions, attributes and behavioural intentions. Worldwide Hospitality and Tourism Themes, v. 2, n. 4, p. 383-397, 2010.

SHEPARD, L. A. The centrality of test use and consequences for test validity. Educational Measurement: Issues and Practice, v. 16, n. 2 , p. 5-24, 1997.

SHONK, D. J.; CHELLADURAI, P. Service Quality, Satisfaction, and Intent to Return in Event Sport Tourism. Journal of Sport Management, v. 22, n. 5, p. 587-602, 2008.

SIRIRAK, S.; ISLAM, N.; KHANG, D. B. Does ICT adoption enhance hotel performance? Journal of Hospitality and Tourism Technology, v. 2, n. 1, p. 34-49, 2011.

SOZUER, A. Self assessment as a gate to performance improvement: A study on hospitality management in Turkey. Procedia Social and Behavioral Sciences, v. 24, n. 0, p. 1090-1097, 2011.

STANCIU, P.; HAPENCIUC, V. Reliability and flexibility in the quality management of tourism products. Amfiteatru Economic, v. 11, n. 26, p. 482-494, 2009.

SVARI, S. et al. A DIP-construct of perceived justice in negative service encounters and complaint handling in the Norwegian tourism industry. Managing Service Quality, v. 20, n. 1, p. 26-45, 2010.

TAPLIN, R. H. The value of self-stated attribute importance to overall satisfaction. Tourism Management, v. 33, n. 2, p. 295-304, Apr 2012.

TEODORESCU, N.; STANCIOIU, A. F.; MITU, A. Considerations regarding quality management in services as a marketing instrument for increasing customers' satisfaction in tourism products. Amfiteatru Economic, v. 11, n. 26, p. 412-418, Jun 2009.

TIEDEMANN, N.; VAN BIRGELE, M.; SEMEIJN, J. Increasing hotel responsiveness to customers through information sharing. Tourism Review, v. 64 , n. 4, p. 12-26, 2009.

TSANG, N. K.-F.; AP, J. Tourists' Perceptions of Relational Quality Service Attributes: A CrossCultural Study. Journal of Travel Research, v. 45, n. 3, p. 355-363, February 1, 2007.

TSAUR, S.-H.; WANG, C.-H. Tip-Collection Strategies, Service Guarantees, and Consumer Evaluations of Group Package Tours. Journal of Travel Research, v. 47, n. 4, p. 523-534, May 1, 2009.

WADONGO, B. et al. Key performance indicators in the Kenyan hospitality industry: A managerial perspective. Benchmarking: An International Journal, v. 17 , n. 6, p. 858-875, 2010.

WALTERS, G.; SPARKS, B.; HERINGTON, C. The Impact of Consumption Vision and Emotion on the Tourism Consumer's Decision Behavior. Journal of Hospitality \& Tourism Research, $p$. 1-24, December 21, 2010.

WANG, F. J.; HUNG, C. J.; LI, P. Y. P. A study on the critical success factors of ISO 22000 implementation in the hotel industry. Pakistan Journal of Statistics, v. 27, n. 5, p. 635-643, 2011.

WANG, K. C. et al. GPTCCC: An instrument for measuring group package tour service. Tourism Management, v. 28, n. 2, p. 361-376, Apr 2007.

WANG, Y.; VELA, M. R.; TYLER, K. Cultural perspectives: Chinese perceptions of UK hotel service quality. International Journal of Culture, Tourism and Hospitality Research, v. 2, n. 4, p. 312-329, 2008.

WEI, M. Analysis of information systems applied to evaluating tourism service quality based on organizational impact. Journal of 
Software, v. 7, n. 3, p. 599-607, 2012.

WEN, I. Factors affecting the online travel buying decision: a review. International Journal of Contemporary Hospitality Management, v. 21, n. 6, p. 752-765, 2009

WOOTEN, M. H.; NORMAN, W. C. Using the ratings grid in tourism/event management. International Journal of Culture, Tourism and Hospitality Research, v. 3, n. 4, p. 347-360, 2009.

WU, J.; SONG, H. Operational performance and benchmarking: A case study of international tourist hotels in Taipei. African Journal of Business Management, v. 5, n. 22, p. 94559465, Sep 2011.

XIAO, Q.; O'NEILL, J. W.; MATTILA, A. S. The role of hotel owners: the influence of corporate strategies on hotel performance. International Journal of Contemporary Hospitality Management, v. 24, n. 1, p. 122-139, 2012.

XIE, X. Service Quality Measurement from Customer Perception Based on Services Science, Management and Engineering. Systems Engineering Procedia, v. 1, n. 0, p. 337343, 2011.

YANG, C. C.; JOU, Y. T.; CHENG, L. Y. Using integrated quality assessment for hotel service quality. Quality \& Quantity, v. 45, n. 2, p. 349364, 2011.

YE, Q.; LI, H.; WANG, Z.; LAW, R. The Influence of Hotel Price on Perceived Service Quality and Value in E-Tourism: An Empirical Investigation Based on Online Traveler Reviews. Journal of Hospitality \& Tourism Research, p. 1096348012442540, April 18, 2012.

YILMAZ, Y.; BITITCI, U. Performance measurement in the valuechain: Manufacturing v. tourism. International Journal of Productivity and Performance Management, v. 55, n. 5, p. 371-389, 2006.

YUAN, Y.-H. E.; WU, C. K. Relationships Among
Experiential Marketing, Experiential Value, and Customer Satisfaction. Journal of Hospitality \& Tourism Research, v. 32, n. 3, p. 387-410, August 1, 2008.

ŽABKAR, V.; BRENČIČ, M. M.; DMITROVIĆ, T. Modelling perceived quality, visitor satisfaction and behavioural intentions at the destination level. Tourism Management, v. 31, n. 4, p. 537546, Aug 2010.

ZOUNI, G.; KOUREMENOS, A. Do Tourism Providers Know their Visitors? An Investigation of Tourism Experience at a Destination. Tourism and Hospitality Research, v. 8, n. 4, p. 282-297, October, 2008. 